\title{
Dual Nature of the Adaptive Immune System in Lampreys
}

\author{
Peng Guo ${ }^{1,{ }^{*}}$, Masayuki Hirano ${ }^{1,{ }^{*}, \text { Brantley R. Herrin }}{ }^{1,{ }^{*}}$, Jianxu Li ${ }^{1}$, Cuiling $\mathrm{Yu}^{1}$, Andrea \\ Sadlonova ${ }^{1}$, and Max D. Cooper ${ }^{1}$ \\ ${ }^{1}$ Emory Vaccine Center and Department of Pathology and Laboratory Medicine, Emory \\ University, 1462 Clifton Road NE, Atlanta, GA 30322, USA
}

\begin{abstract}
Jawless vertebrates use variable lymphocyte receptors (VLR) comprised of leucine-rich-repeat (LRR) segments as counterparts of the immunoglobulin based receptors that jawed vertebrates use for antigen recognition. Highly diverse $V L R$ genes are somatically assembled by the insertion of variable LRR sequences into incomplete germline VLRA and VLRB genes. Here we show that VLRA and VLRB anticipatory receptors are expressed by separate lymphocyte populations through monoallelic VLRA or VLRB assembly in concert with expression of Cytosine deaminase 1 or Cytosine deaminase 2, respectively. Distinctive gene expression profiles for $\mathrm{VLRA}^{+}$and $\mathrm{VLRB}^{+}$lymphocytes resemble those of mammalian T and B cells. Although both VLRA and VLRB cells proliferate in response to antigenic stimulation, only the VLRB lymphocytes bind native antigens and differentiate into VLR antibody secreting cells. Conversely, VLRA lymphocytes respond preferentially to a classical $\mathrm{T}$ cell mitogen and upregulate their expression of proinflammatory cytokine genes, $I L-17$ and $M I F$. The finding of T-like and B-like lymphocytes in lampreys offers new insight into the evolution of adaptive immunity.
\end{abstract}

\begin{abstract}
The ability to mount specific immune responses to a virtually unlimited variety of antigens is apparently unique to vertebrates. The adaptive immune system in jawed vertebrates is characterized by antigen specific cellular and humoral responses, which together provide durable protective immunity 1 . This division of labor is conducted by developmentally separate, but functionally intertwined lineages of clonally diverse lymphocytes, named $\mathrm{T}$ and B cells because they are generated in the thymus or in the avian bursa of Fabricius and mammalian bone marrow2, 3. For antigen recognition, both populations use the same type of immunoglobulin (Ig)-based receptors. The T cell receptors (TCR) and B cell receptors (BCR) are assembled during lymphocyte differentiation by the recombination of different variable (V), diversity (D) and joining (J) immunoglobulin (Ig) gene segments, imprecise $\mathrm{V}$ (D)J splicing, and insertion of non-templated nucleotides at the junctions4-7. The
\end{abstract}

\footnotetext{
Users may view, print, copy, and download text and data-mine the content in such documents, for the purposes of academic research, subject always to the full Conditions of use:http://www.nature.com/authors/editorial_policies/license.html\#terms

Correspondence and requests for materials should be addressed to M.D.C. (max.cooper@emory.edu).

These authors contributed equally to this work.

Author Contributions

P.G., M.H., B.R.H., J.L., C.Y., A.S., M.D.C. designed research, analyzed data and wrote the paper; P.G., M.H., B.R.H., J.L., C.Y., A.S. performed research.

Author Information

The authors declare no competing financial interests.
} 
assembly process is usually completed only on one allele, so that each $\mathrm{T}$ and $\mathrm{B}$ cell expresses a different antigen recognition receptor as the basis for clonal diversity8. Although the $T C R$ and $B C R$ genes are remarkably similar, the antigen binding repertoires of $\mathrm{T}$ and $\mathrm{B}$ cells are very different, primarily because immature $\mathrm{T}$ cells undergo clonal selection on the basis of how well they recognize MHC class I or class II molecules and their self peptide cargos 9-12. This intrathymic selection serves to eliminate $\mathrm{T}$ cells that can recognize and damage self tissues. The ensuing collaboration between $\mathrm{T}$ and $\mathrm{B}$ cells to achieve effective cellular and humoral immunity involves direct cell contact and indirect communication via cytokines and their receptors 1.

When and how this complex adaptive immune system arose are subjects of much conjecture. Even the most basal jawed vertebrates, the cartilaginous sharks, skates and rays, have a thymus in which $\mathrm{T}$ cell progenitors assemble their TCR genes and hematopoietic tissues wherein B cell progenitors assemble their $B C R$ genes13,14. Although the extant jawless vertebrates, lampreys and hagfish, were noted earlier to have lymphocyte-like cells and to produce antigen-specific agglutinins after immunization, neither a recognizable thymus nor $T C R, I g$ and $M H C$ genes have been found15-18. More recent studies indicate that these agnathans generate clonally diverse anticipatory receptors via the assembly of genes for variable lymphocyte receptors (VLR) comprised of leucine-rich-repeat (LRR) subunits and an invariant membrane-proximal stalk region 19. The two germline VLR genes, VLRA and $V L R B$, are incomplete in that they have coding sequences only for portions of the $\mathrm{N}$-terminal LRR and C-terminal LRR subunits and for the stalk region19-23. However, each germline $V L R$ gene is flanked by hundreds of LRR encoding sequences and these are randomly used as templates to add the missing LRR segments needed for completion of a mature $V L R$ gene22. A gene conversion mechanism has been postulated for $V L R$ assembly21, 23, 24, and candidate AID-APOBEC deaminase participants have been identified in the lamprey 21 . $V L R B$ assembly has been shown to be confined to one allele19, 23, ensuring that each VLRB lymphocyte expresses a receptor of unique sequence, but it is not yet known whether the same is true for VLRA.

In earlier studies, we found that the VLRB bearing lymphocytes resemble B lymphocytes of jawed vertebrates. They can bind bacterial or erythrocyte antigens and respond by proliferation and differentiation into plasmacytes that secrete multimeric VLRB antibodies specific for either protein or carbohydrate epitopes25, 26. In this study, we used VLRA specific antibodies to identify a second population of lamprey lymphocytes and to determine their VLR gene assembly status, gene expression profile, tissue distribution, morphology and functional responses to antigens and mitogens in comparison with the VLRB lymphocytes. Our findings indicate that VLRA lymphocytes are surprisingly similar to the T cells in jawed vertebrates, and suggest functional interactions between the VLRA and VLRB lymphocyte populations.

\section{Identification of VLRA+ and VLRB+ lymphocyte populations}

The invariant stalk regions of the lamprey VLRA and VLRB proteins share less than $11 \%$ amino acid sequence identity, a difference that allowed the production of VLRB specific monoclonal antibodies for use in characterizing the VLRB bearing lymphocytes 22,25 . In 
order to identify VLRA bearing lymphocytes, we used a VLRA-Ig Fc fusion protein as an immunogen to produce two rabbit antisera and three mouse monoclonal antibodies. The VLRA specificity of the antibody preparations was established by comparative analysis with a VLRB monoclonal antibody in ELISA and immunoblot assays (Supplementary Fig. 1). Using these VLR specific antibodies for immunofluorescence staining assays, we could identify discrete $\mathrm{VLRA}^{+}$and $\mathrm{VLRB}^{+}$lymphocyte populations in the principal lymphoid tissues of lamprey larvae, the blood, kidneys, typhlosole and gill region (Fig. 1a). VLRA bearing lymphocytes were detected in the same relative frequencies by each of the VLRA specific reagents (Supplementary Fig. 2). Notably, the VLRB ${ }^{+}$lymphocytes significantly outnumbered VLRA ${ }^{+}$lymphocytes in all tissues, except the gill region. The VLRB predominance was most striking in blood and kidneys, wherein the VLRB to VLRA ratio was approximately 8 to 1 (Fig. 1b); examination of adult blood samples indicated persistent VLRB predominance. These findings indicate that VLRA- and VLRB-bearing cells belong to separate lymphocyte populations that are dispersed through the body in a characteristic tissue distribution pattern.

\section{Genetic basis of VLRA versus VLRB expression}

To explore the genetic basis for the differential expression of VLR types by the two lymphocyte populations, we used fluorescence activated cell sorting to purify VLRA ${ }^{+}$, $\mathrm{VLRB}^{+}$and VLRA/VLRB double-negative (DN) populations of cells having the light scatter characteristics of lymphocytes. The VLRA ${ }^{+}$and $\mathrm{VLRB}^{+}$lymphocytes could be separated cleanly ( $>95 \%$ purity), whereas the DN population included several cell types, among which nucleated thrombocytes constituted the vast majority. When VLR transcription was evaluated for each population by quantitative PCR analysis, the VLRA ${ }^{+}$ lymphocytes expressed VLRA transcripts exclusively, VLRB ${ }^{+}$lymphocytes expressed VLRB transcripts exclusively, and the DN population expressed few or no VLR transcripts of either type. A representative experiment illustrating these results for adult blood cells is shown in Fig. 1c, and the same pattern of selective VLRA and VLRB expression was observed for the sorted populations of blood, kidney and typhlosole cells from larval stage lampreys three to four years of age (Supplementary Fig. 3).

When VLRA and VLRB gene configurations were examined for the $\mathrm{VLRA}^{+}, \mathrm{VLRB}^{+}$and DN cells using paired 5' and 3' PCR primers (Fig. 2a and b), we observed mature VLRA assembly only in the VLRA ${ }^{+}$lymphocyte population and the same relationship held for $V L R B$ assembly by $\mathrm{VLRB}^{+}$lymphocytes. Mature and germline VLRA PCR products were observed in equal proportions (Supplementary Fig. 4), indicating that $V L R A$ assembly occurs on one allele, a finding that parallels the monoallelic pattern of VLRB assembly demonstrated previously19, 23. Because the lamprey AID-like deaminases have been postulated to serve a role in the gene conversion mechanism postulated for $V L R$ gene assembly21, we examined the expression of $C D A 1$ and $C D A 2$ in the sorted cell populations. Remarkably, CDAl expression was observed only in VLRA ${ }^{+}$cells, while $C D A 2$ expression was limited to the $\mathrm{VLRB}^{+}$population (Fig. 2c). These findings suggest that the monoallelic $V L R A$ versus $V L R B$ gene assembly in the two lymphocyte populations is linked to expression of the genes for the CDA1 or CDA2 deaminases, respectively. 


\section{Distinctive gene expression profiles}

Examination of the $\mathrm{VLRA}^{+}, \mathrm{VLRB}^{+}$and $\mathrm{DN}$ populations for expression of a selected panel of genes indicated they have different gene expression profiles, and the distinctions were particularly informative for the two lymphocyte populations. The discriminating genes expressed by VLRA and VLRB lymphocyte populations included ones for transcription factors, Notch1, cytokine/chemokines and their receptors, Toll-like receptors (TLR) and various signaling molecules (Fig. 3, Supplementary Tables $1-3$ and Supplementary Fig. 5). A surprising number of the genes that were selectively expressed by VLRA lymphocytes are orthologs of genes that are typically, although not exclusively, expressed by $\mathrm{T}$ lineage cells in jawed vertebrates. The genes expressed preferentially by VLRA lymphocytes included several transcription factors that may be used for T cell differentiation, GATA2/327, 28, cRel29, aryl hydrocarbon receptor (AHR)30 and BCL11b31, a chemokine receptor involved in lymphocyte progenitor homing to the thymus, CCR932, the T cell fate determining molecule, Notch133, a receptor protein tyrosine phosphatase that is essential for $\mathrm{T}$ cell development, CD4534, the receptor for interleukin 8 (IL-8), CXCR235, and two proinflammatory cytokines made by T cells, IL-1736, 37 and macrophage migration inhibitory factor (MIF)38, 39. The contrasting gene expression profile noted for the VLRB population included genes for the hematopoietic progenitor homing receptor, CXCR440, the herpes virus entry molecule (TNFR 14) which binds to LIGHT on T cells41, two components of the BCR mediated signaling cascade, Syk42and the B cell adaptor protein (BCAP) 43 , an Ig superfamily member with inhibitory potential, TCRL44, the chemotactic inflammatory cytokine, IL-845, the receptor for IL-1736, and the Toll-like receptor orthologs, TLR2abc, TLR7 and TLR10, the ligation of which is important for B cell activation46-48. This analysis, albeit limited by the incomplete status of the lamprey genomic sequence analysis, reveals gene expression profiles for VLRA and VLRB lymphocytes that are surprisingly similar to those for $\mathrm{T}$ and $\mathrm{B}$ cells in jawed vertebrates.

\section{VLRA and VLRB lymphocyte responses to antigens}

In response to immunization with Bacillus anthracis exosporium, antigen-binding VLRB ${ }^{+}$ members of the lymphocyte population undergo proliferation and differentiation into plasmacytes that secrete multivalent VLRB antibodies against BclA, a major protein in the outermost layer of $B$. anthracis spores 25 . In the present experiments, we found that both the VLRA and VLRB lymphocyte populations responded to this antigen with comparable levels of proliferation (Fig. 4a). In contrast, VLRA lymphocytes that could bind anthrax spores were not demonstrable either before or after immunization (Fig. 4b); the same paradoxical results were obtained when Escherichia coli binding by VLRA and VLRB lymphocyte was examined before and after immunization (data not shown). Equally surprising, the VLRA population failed to secrete VLRA proteins that could bind B. anthracis, E. coli, Salmonella typhimurium, or Streptococcus pneumoniae following immunizations that led to the secretion of specific VLRB antibodies for each of these bacteria (Fig. 4c and Supplementary Fig. 6). In addition, VLRA proteins could not be detected in lamprey plasma by immunoblot analysis (Fig. 4d and Supplementary Fig. 7). 
To determine whether an inherent difference in the two types of receptors could explain the failure to secrete VLRA, we transfected cells of the 293T human embryonic kidney cell line with either VLRA or VLRB cDNAs to compare their patterns of intracellular synthesis and secretion. While the VLRB transfected cells secreted their VLRB protein products in the multimeric forms described previously26, the VLRA transfected cells expressed their VLRA protein products on the cell surface, but did not secrete them (Supplementary Fig. 8). These results indicate that VLRA lymphocytes respond to antigenic stimulation in a manner very reminiscent of the $T$ cell response and confirm that VLRB lymphocytes respond to antigens much like B lymphocytes. In addition, the contrast between the abilities of VLRA and VLRB lymphocytes to bind native bacterial surface epitopes strongly suggests they have different receptor repertoires.

\section{Preferential PHA responsiveness of VLRA lymphocytes}

Because of the resemblance between the VLRA lymphocytes and T lymphocytes in jawed vertebrates, we compared the responses of VLRA and VLRB lymphocytes to stimulation by the plant lectin, phytohemagglutinin (PHA), a classical T cell mitogen. When larvae were injected with PHA, the VLRA ${ }^{+}$lymphocytes responded vigorously by undergoing lymphoblastoid transformation and proliferation to become the predominant lymphocyte population (Fig. 5a and b). The activated VLRA ${ }^{+}$cells were found by transmission EM to be large lymphoblasts with much less rough endoplasmic reticulum than the previously described VLRB secreting plasmacytes (Fig. 5c)25. While VLRB lymphocytes also responded to PHA stimulation, their response was much weaker in terms of cell size increase and numbers of proliferating cells in this population. Nevertheless, PHA stimulation enhanced VLRB cell expression of IL-8 transcripts, in contrast to its enhancement of VLRA cell expression of IL-17 and MIF transcripts (Fig. 5d). These findings, coupled with the reciprocal expression of $I L-8 R$ by VLRA lymphocytes and $I L-17 R$ expression by VLRB lymphocytes (Fig. 3), are indicative of the potential for functional interactions between the two lymphocyte populations.

\section{Discussion}

This unforeseen genotypic and functional division of lymphocyte differentiation in lampreys offers an intriguing new piece to the puzzle of how the adaptive immune systems may have evolved. Our initial definition of the VLRA and VLRB lymphocyte populations also offers interesting clues to presently unresolved issues regarding their differentiation and function.

One question that is immediately raised by our findings concerns the anatomical location(s) in which VLRA lymphocytes begin their differentiation. Indeed, one of the reasons why the remarkable resemblance of VLRA lymphocytes to T lymphocytes was so surprising is the thus far fruitless quest to identify a thymus equivalent in lampreys16, 17. However, the relatively high concentration of VLRA lymphocytes in the gill region could favor the branchial region as a potential site for their generation. In addition, our gene profile analysis indicates that VLRA lymphocytes express an array of transcription factors, chemokine receptors and Notch1 that lymphocyte progenitors use for homing to the thymus and $\mathrm{T}$ lineage commitment in jawed vertebrates. Analysis of the expression of the corresponding 
chemokines and Notch1 ligands in the pharyngeal pouch-derived epithelial cells and the neighboring VLRA lymphocytes could therefore facilitate the identification of a thymus equivalent in lampreys.

Solving the issue of where the VLRA and VLRB genes are assembled will also require better understanding of the molecular mechanisms involved in this process. The donor and recipient $L R R$ sequences used in $V L R$ assembly lack recombination signal sequences that characterize the Ig V, D and J gene segments, and the Rag1 and Rag2 recombination activation genes employed for $T C R$ and $B C R$ gene assembly have not been found in lampreys18. The presently known features of $V L R$ gene assembly instead suggest that a gene conversion mechanism is used to copy the donor LRR sequences21, 23, 24. In accord with this hypothesis, two APOBEC-AID family orthologs, $C D A 1$ and $C D A 2$, have been identified in the lamprey. CDA1 has been shown to have an AID-like DNA deaminase mutator function in bacteria and yeast lacking uracil DNA glycosylase activity21.

Although the functional competency of CDA2 has not yet been elaborated, it shares the DNA deaminase catalytic motif with CDA1 and has in addition an extended C-terminus containing a small AT-hook peptide motif that may confer DNA binding specificity. These distinctive features and the restriction of CDA1 expression to the VLRA lymphocyte population and CDA2 expression to VLRB lymphocytes thus imply the potential for selective involvement of these enzymes in VLRA and VLRB assembly during lymphocyte development.

One of the most provocative issues raised by our findings concerns the mode of antigen recognition by VLRA bearing lymphocytes. We expected to find comparable antigen binding and response patterns for the two lymphocyte populations, since the VLRA and VLRB proteins are very similar and have comparable diversity. The proliferative responses of VLRA and VLRB lymphocytes to immunization indeed were found to be equivalent. Surprisingly though, antigen-binding VLRA lymphocytes could not be found before or after immunization, whereas specific antigen-binding VLRB lymphocytes were easily detected in naive animals and immunization increased their frequency. One possible explanation for the inability of VLRA lymphocytes to bind native epitopes on immunogens that induce their proliferation is that VLRA lymphocytes recognize processed antigens and undergo selection in a manner analogous to the $\mathrm{T}$ lymphocyte repertoire selection in jawed vertebrates. Lampreys lack the major histocompatibility complex genes for MHC class I and class II molecules that accessory cells use to present peptide fragments to $\mathrm{T}$ cells in jawed vertebrates 18. However, a precedent for an alternative mechanism for self recognition is provided by the recent identification of a highly polymorphic fusion/histocompatibility gene locus in Botryllus schlosseri49, a colonial tunicate member of a vertebrate sister group in the new chordate phylogeny50. The nature of the antigens recognized by VLRA anticipatory receptors presently remains an unresolved enigma.

The failure of VLRA cells to secrete their receptors as soluble proteins was the most striking functional difference observed for the VLRA and VLRB lymphocytes. This feature could reflect an inability of VLRA cells to develop the necessary secretory machinery. However, when VLRA proteins were produced by a human cell line that can secrete VLRB proteins, 
the transfected cells produced cell surface VLRA proteins, but did not secrete them. The VLRA proteins, like the TCR proteins, thus appear to be expressed exclusively as transmembrane molecules. The preferential expression of TLR2, TLR7 and TLR10 orthologs by the VLRB lymphocytes suggests that TLR ligands may facilitate activation of this population of lymphocytes in a manner similar to their roles in activating B lymphocytes. The development of appropriate conditions for culturing lamprey lymphocytes and cell lines will facilitate the future analysis of the signaling mechanisms employed by VLRA and VLRB lymphocytes.

Many of the elaborate cytokine networks that $\mathrm{T}$ and $\mathrm{B}$ cells use to coordinate effective inflammatory responses in jawed vertebrates are not evident in the more basal jawless vertebrates. Agnathan orthologs have not been identified for the interferons and interferon receptors, IL-2 / IL-2R, IL-4 / IL4R, IL-7 / IL-7R and other cytokine/cytokine receptor pairs. Nevertheless, the VLRA lymphocytes preferentially express IL-17 and MIF transcripts and their enhanced expression after activation suggests that VLRA lymphocytes may use these pro-inflammatory cytokines to engage the IL-17R bearing VLRB lymphocytes for defense purposes, in a manner similar to their $\mathrm{T}$ cell counterparts. Conversely, activated VLRB lymphocytes upregulate their expression of IL-8 transcripts, suggesting they may use this cytokine to attract and engage IL-8R bearing VLRA lymphocytes and other cell types to amplify the response to potential pathogens.

The evolution around 500 million years ago of two very different anticipatory receptors of comparable diversity in jawless and jawed vertebrates, while conserving similar compartmentalization of lymphocyte differentiation, strongly attests the survival value of adaptive immunity. The evolution of an LRR-based adaptive immune system in lampreys and hagfish, apparently before the second round of whole genomic replication, and an Igbased adaptive immune system in jawed vertebrates, after a second round of genomic duplication50, suggests that these very different types of anticipatory receptors were convergent evolutionary acquisitions 19 . The failure so far to find relics of the VLR genes in jawed vertebrates is also consistent with an independent evolution of clonally diverse LRRbased receptors versus Ig-based receptors for otherwise similar adaptive immune systems. An obvious advantage of adaptive immunity over the elaborate invertebrate strategies for innate immunity is the capacity for memory of previous encounters with infectious agents. On the other hand, the development of a randomly generated anticipatory repertoire of great diversity inevitably creates the hazard of self-reactivity and attendant autoimmunity. The orchestration of cellular and humoral immune responses by T cells, whose TCR repertoire is shaped during development to discriminate between self and non-self antigens, provides an important safeguard against autoimmunity in jawed vertebrates. The potential for autoimmunity theoretically would necessitate a similar solution for the adaptive immune system in jawless vertebrates. Our findings thus suggest that dual recognition and response arms with intertwined function were fundamental to the evolution of adaptive immunity in vertebrates. 


\section{Methods Summary}

Sea lamprey larvae ( $8-15 \mathrm{~cm}, 2-4$ years of age) were collected from tributaries to Lake Michigan (Lamprey services, Michigan) and housed in sand-lined aquariums at $20^{\circ} \mathrm{C}$. Larvae anesthetized with $0.1 \mathrm{~g} / \mathrm{l} \mathrm{MS222} \mathrm{(Sigma)} \mathrm{were} \mathrm{given} \mathrm{intracoelomic} \mathrm{injections} \mathrm{of}$ antigens or mitogens ( $25 \mu \mathrm{g}$ phytohemaglutinin (PHA)-L (Sigma)) prepared in $60 \mu \mathrm{l}$ of sterile $0.67 \mathrm{X}$ PBS. Injections were administered on days 0 and 14, and tissues collected from lampreys euthanized with $1 \mathrm{~g} / \mathrm{l}$ MS222 on day 28 (unless otherwise indicated). Blood was collected in $30 \mathrm{mM}$ EDTA/0.67X PBS. Buffy coat leukocytes were separated from red blood cells by centrifugation at $50 \times \mathrm{g}$. Leukocytes were isolated from kidney, typhlosole, and gills by disrupting tissues between frosted glass slides.

For VLRA antibody production, four VLRA cDNAs isolated from lamprey lymphocytes were cloned in-frame with the constant region of human IgG1 (IgG1-Fc). The VLRA-IgG1Fc fusion proteins were expressed in HEK-293T cells and purified from tissue culture supernatants by protein A (GE Healthcare) chromatography. Monoclonal anti-VLRA antibodies were produced by immunization of BALB/c mice with VLRA-IgG1-Fc proteins emulsified in TiterMax Gold adjuvant. Lymphocytes from the draining lymph nodes of immunized mice were fused with the Ag8.653 myeloma cell line using PEG-1500 (Roche). Three VLRA-specific hybridoma clones (9A6 (IgG1), 9B3 (IgG1) and 2A5 (IgG1)) were identified by ELISA with VLRA recombinant protein and immunoblotting of VLRA transfectants. Anti-VLRA polyclonal antisera were produced by immunization of rabbits with the VLRA-IgG1-Fc proteins (PickCell Laboratories BV, Netherlands).

\section{Methods}

Antigens

Escherichia coli (ATCC 25922 strain), Salmonella enterica Typhimurium (WB99-SR11 strain), and Streptococcus pneumoniae (R36A strain) were provided by David E. Briles and William H. Benjamin, Jr. (University of Alabama at Birmingham). Bacteria were heat-killed by incubation at $60^{\circ} \mathrm{C}$ for 1 hour. $5 \times 10^{8}$ heat-killed E. coli, S. typhimurium, and $1 \times 10^{8} \mathrm{~S}$. pneumoniae were used for immunization. Bacillus anthracis (spores and exosporium) and Bacillus cereus spores were provided by Charles L. Turnbough Jr. (University of Alabama at Birmingham). Lampreys were immunized with $10 \mu \mathrm{g}$ of B. anthracis exosporium.

\section{Flow cytometric analysis of VLRA and VLRB lymphocytes}

Leukocytes from blood and tissues were stained with anti-VLRA rabbit polyclonal serum (R110 or R111) or mouse mAbs (9A6, 9B3, 2A5, IgG1) and anti-VLRB mouse mAb (4C4, $\operatorname{IgG} 2 b$ ) for 10 minutes on ice, washed twice and stained with fluorescent-conjugated secondary Abs. For anti-VLRA mAb (IgG1) and anti-VLRB mAb (4C4, IgG2b) staining combinations, R-phycoerythrin (PE)-conjugated goat anti-mouse IgG1 Abs (Southern Biotech) and FITC-conjugated goat anti-mouse IgG2b Abs (Southern biotech) were used. When staining with anti-VLRA rabbit serum and anti-VLRB mAb (4C4), RPE-conjugated goat anti-rabbit Ig Abs (Southern biotech) and allophycocyanin (APC)-conjugated goat anti- 
mouse Ig Abs (Southern Biotech) were used. Cells were analyzed on a CyAn ADP highperformance flow cytometer (Dako). Staining and washes were in 0.67X PBS with 1\% BSA.

\section{Immunoblotting}

Samples were separated on $10 \%$ SDS-PAGE gels with or without $5 \%$ (vol/vol) 2mercaptoethanol before transfer onto nitrocellulose membranes. Membranes were blocked overnight with $5 \%$ milk and incubated with anti-VLRA or anti-VLRB antibodies for one hour. After 5 washes with PBS-0.5\% Tween-20, membranes were incubated with HRPconjugated goat anti-rabbit or mouse Ig polyclonal Abs (Southern Biotech) and washed. Blots were developed using SuperSignal West Pico chemiluminescent substrate (Pierce, Rockford, IL).

\section{Genomic DNA and PCR}

Genomic DNA was extracted from VLRA ${ }^{+}, \mathrm{VLRB}^{+}$and VLR double negative-sorted cells in blood and tissue lymphocytes using DNeasy kit (QIAGEN). Genomic PCR was carried out using primers VLRA-F + VLRA-R (Expand High Fidelity, Roche and Ex Taq, Takara) or VLRB-F + VLRB-R (Expand Long Template, Roche). Primers are listed in Supplementary Table 1.

\section{Quantitative PCR}

Target gene sequences were obtained from the National Center for Biotechnology Information database or the lamprey genome database of the Genome Sequencing Center at Washington University. Cells in the lymphocyte-gate were sorted into $\mathrm{VLRA}^{+}, \mathrm{VLRB}^{+}$and VLR double negative populations on a BD FACS Aria II. RNA was extracted from each population using RNeasy kit with on-column DNA digestion by DNase I (QIAGEN). Firststrand cDNA was synthesized with random hexamer primers and Superscript III (Invitrogen). Quantitative real-time PCR (qPCR) was done with SYBR Green on a 7900HT ABI Prism (Applied Biosystems). Three independent qPCR reactions were performed at different times for each sample. The value of the target gene was normalized to $\beta$-actin. The normalized value of each gene was compiled into a heat map ( $z$-score $x$ 10). Magenta: $z>0$, green: $\mathrm{z}<0$ and yellow: $\mathrm{z}=0[\mathrm{z}=$ (each value - average)/standard deviation $]$. Primers are described in Supplementary Table 1.

\section{Proliferation Assay}

Lampreys stimulated with antigen or mitogen were injected with $5 \mu \mathrm{g}$ of 5-ethynyl-2'deoxyuridine (EdU) (Invitrogen) in $60 \mu \mathrm{l} 0.67 \mathrm{X}$ PBS and returned to their tanks for $24 \mathrm{hrs}$ before collection of leukocytes for staining with anti-VLRA or anti-VLRB Abs. Stained cells were fixed in $4 \%$ paraformaldehyde for $15 \mathrm{~min}$ at room temperature, then resuspended in $100 \mu \mathrm{L}$ of the $1 \mathrm{X}$ saponin-based permeabilization and wash buffer for $10 \mathrm{~min}$ on ice. $\mathrm{EdU}^{+}$cell detection was performed according to the manufacturer's protocol (Click-iT ${ }^{\mathrm{TM}}$ EdU Alexa Fluor 488 Flow Cytometry Assay Kit, Invitrogen). Briefly, cells were incubated with the EdU reaction cocktail, which contains Alexa Fluor 488-azide for $30 \mathrm{~min}$ at room temperature, washed twice in $1 \mathrm{X}$ saponin-based permeabilization and wash buffer, and analyzed by flow cytometry. 
ELISA

for animals immunized with $B$. anthracis exosporium, $10^{6}$ B. anthracis spores or B. cereus strain $\mathrm{T}$ spores per well were applied on poly-L-lysine-coated plates as per Alder MN et al 22. For animals immunized with bacteria, $5 \times 10^{6}$ heat-killed bacteria per well were applied on poly-L-lysine-coated plates. Plates were blocked with $1 \%$ BSA in PBS for $1 \mathrm{hr}$. Seriallydiluted plasma was applied for 2 hours at $37^{\circ} \mathrm{C}$. VLRs were detected with anti-VLRB (4C4) or anti-VLRA (9A6) mAb and alkaline phosphatase-conjugated goat anti-mouse Ig Abs (Southern Biotech). ELISAs were developed with phosphatase substrate (Sigma) and read at $405 \mathrm{~nm}$ (Versamax microplate reader; Molecular Devices, Sunnyvale, CA).

\section{Lymphocyte antigen-binding assay}

$10^{6}$ lamprey leukocytes were incubated with $10^{6}$ Alexa Fluor 488-labeled spores or $10^{7}$ Alexa Fluor 488-labeled bacteria for $20 \mathrm{~min}$ on ice. Spores and bacteria were labeled using the Alexa Fluor 488 Protein Labeling Kit (Invitrogen). Cells were stained with anti-VLRA (R110) and anti-VLRB (4C4) Abs followed by APC-conjugated goat anti-mouse Ig Abs (Southern Biotech) and R-PE-conjugated goat anti-rabbit Ig Abs (Southern Biotech), then analyzed by flow cytometry.

\section{VLR expression in HEK-293T cells}

VLRA and VLRB plasmids were transfected into HEK-293T cells cultured in DMEM containing 5\% fetal bovine serum (FBS) using linear polyethylenimine (PEI), MW 25,000 (Polysciences, Inc.) at a 3:1 PEI to DNA ratio. Cells were separated from supernatants $48 \mathrm{~h}$ post-transfection by centrifugation at $300 \times \mathrm{g}$ and lysed in $1 \%$ NP-40 lysis buffer.

\section{Lymphocyte counts}

Lymphocytes were extracted from lamprey kidney and typhlosole by passage through a 70 $\mu \mathrm{m}$ cell strainer (BD Bioscience) to obtain a single cell suspension. Cells were washed and resuspended in $1 \mathrm{ml} 0.67 \mathrm{X}$ PBS. Total cells were counted in $25 \mu \mathrm{l}$ of each sample on an Accuri C6 flow cytometer (Accuri Cytometers Inc, Michigan). The total number of cells in the 'lymphocyte gate' was calculated by the following formula: total lymphocyte number = number of cells in the lymphocytes gate $\times 40$ (dilution factor: $1000 \mu \mathrm{l} / 25 \mu \mathrm{l}$ ). Lymphocytes were then stained with anti-VLRA and VLRB Abs to determine the percentage of VLRA ${ }^{+}$ and $\mathrm{VLRB}^{+}$cells in the samples. These percentages were used to calculate the total number of lymphocytes of each type.

\section{Electron microscopy}

Blood lymphocytes from naïve or PHA stimulated lamprey were sorted on BD FACS Aria II (BD Bioscience). The VLRA-positive cells were prepared for transmission electron microscopic analysis as described as per Alder MN et al 22.

\section{Statistical Analysis}

A two-sample Student's t-test was used for statistical analysis. 


\section{Acknowledgements}

We thank Matthew N. Alder and Götz R. A. Ehrhardt for helpful suggestions and discussion; Charles L. Turnbough, Jr. for providing B. anthracis spores and exosporium; David E. Briles and William H. Benjamin, Jr. for E. coli, S. pneumoniae, and S. typhimurium; Hong Yi for help with electron microscopy; Sommer A. Durham and Robert E. Karaffa, II for help with cell sorting; Marsha Flurry for help with preparation of figures. This work is supported by National Institutes of Health and Georgia Research Alliance.

\section{References}

1. Paul, WE. Fundamental Immunology. Sixth Edition. Philadelphia: Lippincott Williams \& Wilkins; 2008.

2. Cooper MD, Peterson RD, Good RA. Delineation of the Thymic and Bursal Lymphoid Systems in the Chicken. Nature. 1965; 205:143-146. [PubMed: 14276257]

3. Roitt IM, Greaves MF, Torrigiani G, Brostoff J, Playfair JH. The cellular basis of immunological responses. A synthesis of some current views. Lancet. 1969; 2:367-371. [PubMed: 4183985]

4. Tonegawa S. Somatic generation of antibody diversity. Nature. 1983; 302:575-581. [PubMed: 6300689]

5. Yanagi Y, et al. A human T cell-specific cDNA clone encodes a protein having extensive homology to immunoglobulin chains. Nature. 1984; 308:145-149. [PubMed: 6336315]

6. Hedrick SM, Cohen DI, Nielsen EA, Davis MM. Isolation of cDNA clones encoding T cell-specific membrane-associated proteins. Nature. 1984; 308:149-153. [PubMed: 6199676]

7. Jung D, Giallourakis C, Mostoslavsky R, Alt FW. Mechanism and control of V(D)J recombination at the immunoglobulin heavy chain locus. Annu. Rev. Immunol. 2006; 24:541-570. [PubMed: 16551259]

8. Burnet FM. A modification of Jerne's theory of antibody production using the concept of clonal selection. Aust. J. Sci. 1957; 20:67-69.

9. Zinkernagel RM, Doherty PC. Cytotoxic thymus-derived lymphocytes in cerebrospinal fluid of mice with lymphocytic choriomeningitis. J. Exp. Med. 1973; 138:1266-1269. [PubMed: 4542807]

10. Nikolic-Zugic J, Bevan MJ. Role of self-peptides in positively selecting the T-cell repertoire. Nature. 1990; 344:65-67. [PubMed: 2304556]

11. von Boehmer H. Selection of the T-cell repertoire: receptor-controlled checkpoints in T-cell development. Adv. Immunol. 2004; 84:201-238. [PubMed: 15246254]

12. Unanue ER. Perspective on antigen processing and presentation. Immunol. Rev. 2002; 185:86102. [PubMed: 12190925]

13. Flajnik MF. Comparative analyses of immunoglobulin genes: surprises and portents. Nat. Rev. Immunol. 2002; 2:688-698. [PubMed: 12209137]

14. Litman GW, Cannon JP, Dishaw LJ. Reconstructing immune phylogeny: new perspectives. Nat. Rev. Immunol. 2005; 5:866-879. [PubMed: 16261174]

15. Finstad J, Good RA. The Evolution of the Immune Response. 3. Immunologic Responses in the Lamprey. J. Exp. Med. 1964; 120:1151-1168. [PubMed: 14238932]

16. Ardavin CF, Zapata A. The pharyngeal lymphoid tissue of lampreys. A morpho-functional equivalent of the vertebrate thymus? Thymus. 1988; 11:59-65. [PubMed: 3344544]

17. Boehm T, Bleul CC. The evolutionary history of lymphoid organs. Nat. Immunol. 2007; 8:131135. [PubMed: 17242686]

18. Uinuk-Ool T, et al. Lamprey lymphocyte-like cells express homologs of genes involved in immunologically relevant activities of mammalian lymphocytes. Proc. Natl. Acad. Sci. U S A. 2002; 99:14356-14361. [PubMed: 12391333]

19. Pancer Z, et al. Somatic diversification of variable lymphocyte receptors in the agnathan sea lamprey. Nature. 2004; 430:174-180. [PubMed: 15241406]

20. Pancer Z, et al. Variable lymphocyte receptors in hagfish. Proc. Natl. Acad. Sci. U S A. 2005; 102:9224-9229. [PubMed: 15964979] 
21. Rogozin IB, et al. Evolution and diversification of lamprey antigen receptors: evidence for involvement of an AID-APOBEC family cytosine deaminase. Nat. Immunol. 2007; 8:647-656. [PubMed: 17468760]

22. Alder MN, et al. Diversity and function of adaptive immune receptors in a jawless vertebrate. Science. 2005; 310:1970-1973. [PubMed: 16373579]

23. Nagawa F, et al. Antigen-receptor genes of the agnathan lamprey are assembled by a process involving copy choice. Nat. Immunol. 2007; 8:206-213. [PubMed: 17187071]

24. Cooper MD, Alder MN. The evolution of adaptive immune systems. Cell. 2006; 124:815-822. [PubMed: 16497590]

25. Alder MN, et al. Antibody responses of variable lymphocyte receptors in the lamprey. Nat. Immunol. 2008; 9:319-327. [PubMed: 18246071]

26. Herrin BR, et al. Structure and specificity of lamprey monoclonal antibodies. Proc. Natl. Acad. Sci. U S A. 2008; 105:2040-2045. [PubMed: 18238899]

27. Ho IC, Tai TS, Pai SY. GATA3 and the T-cell lineage: essential functions before and after Thelper-2-cell differentiation. Nat. Rev. Immunol. 2009; 9:125-135. [PubMed: 19151747]

28. Suzuki T, Shin IT, Kohara Y, Kasahara M. Transcriptome analysis of hagfish leukocytes: a framework for understanding the immune system of jawless fishes. Dev. Comp. Immunol. 2004; 28:993-1003. [PubMed: 15236930]

29. Banerjee D, Liou HC, Sen R. c-Rel-dependent priming of naive T cells by inflammatory cytokines. Immunity. 2005; 23:445-458. [PubMed: 16226509]

30. Quintana FJ, et al. Control of T(reg) and $\mathrm{T}(\mathrm{H}) 17$ cell differentiation by the aryl hydrocarbon receptor. Nature. 2008; 453:65-71. [PubMed: 18362915]

31. Wakabayashi Y, et al. Bcl11b is required for differentiation and survival of alphabeta $\mathrm{T}$ lymphocytes. Nat. Immunol. 2003; 4:533-539. [PubMed: 12717433]

32. Liu C, et al. Coordination between CCR7-and CCR9-mediated chemokine signals in prevascular fetal thymus colonization. Blood. 2006; 108:2531-2539. [PubMed: 16809609]

33. Maillard I, Fang T, Pear WS. Regulation of lymphoid development, differentiation, and function by the Notch pathway. Annu. Rev. Immunol. 2005; 23:945-974. [PubMed: 15771590]

34. Kishihara K, et al. Normal B lymphocyte development but impaired T cell maturation in CD45exon6 protein tyrosine phosphatase-deficient mice. Cell. 1993; 74:143-156. [PubMed: 8334701]

35. Lippert U, Zachmann K, Henz BM, Neumann C. Human T lymphocytes and mast cells differentially express and regulate extra-and intracellular CXCR1 and CXCR2. Exp. Dermatol. 2004; 13:520-525. [PubMed: 15265017]

36. Weaver CT, Hatton RD, Mangan PR, Harrington LE. IL-17 family cytokines and the expanding diversity of effector T cell lineages. Annu. Rev. Immunol. 2007; 25:821-852. [PubMed: 17201677]

37. Tsutsui S, Nakamura O, Watanabe T. Lamprey (Lethenteron japonicum) IL-17 upregulated by LPS-stimulation in the skin cells. Immunogenetics. 2007; 59:873-882. [PubMed: 17924104]

38. Bloom BR, Shevach E. Requirement for T cells in the production of migration inhibitory factor. J. Exp. Med. 1975; 142:1306-1311. [PubMed: 1104742]

39. Weiser WY, et al. Molecular cloning of a cDNA encoding a human macrophage migration inhibitory factor. Proc. Natl. Acad. Sci. U S A. 1989; 86:7522-7526. [PubMed: 2552447]

40. Ma Q, Jones D, Springer TA. The chemokine receptor CXCR4 is required for the retention of B lineage and granulocytic precursors within the bone marrow microenvironment. Immunity. 1999; 10:463-471. [PubMed: 10229189]

41. Murphy KM, Nelson CA, Sedy JR. Balancing co-stimulation and inhibition with BTLA and HVEM. Nat. Rev. Immunol. 2006; 6:671-681. [PubMed: 16932752]

42. Jumaa H, Hendriks RW, Reth M. B cell signaling and tumorigenesis. Annu. Rev. Immunol. 2005; 23:415-445. [PubMed: 15771577]

43. Okada T, Maeda A, Iwamatsu A, Gotoh K, Kurosaki T. BCAP: the tyrosine kinase substrate that connects B cell receptor to phosphoinositide 3-kinase activation. Immunity. 2000; 13:817-827. [PubMed: 11163197] 
44. Yu C, Ehrhardt GR, Alder MN, Cooper MD, Xu A. Inhibitory signaling potential of a TCR-like molecule in lamprey. Eur. J. Immunol. 2009; 39:571-579. [PubMed: 19130486]

45. Sims-Mourtada JC, et al. In vivo expression of interleukin-8, and regulated on activation, normal, T-cell expressed, and secreted, by human germinal centre B lymphocytes. Immunology. 2003; 110:296-303. [PubMed: 14632656]

46. Pasare C, Medzhitov R. Control of B-cell responses by Toll-like receptors. Nature. 2005; 438:364368. [PubMed: 16292312]

47. Akira S, Uematsu S, Takeuchi O. Pathogen recognition and innate immunity. Cell. 2006; 124:783801. [PubMed: 16497588]

48. Ishii A, et al. Lamprey TLRs with properties distinct from those of the variable lymphocyte receptors. J. Immunol. 2007; 178:397-406. [PubMed: 17182578]

49. De Tomaso AW, et al. Isolation and characterization of a protochordate histocompatibility locus. Nature. 2005; 438:454-459. [PubMed: 16306984]

50. Putnam NH, et al. The amphioxus genome and the evolution of the chordate karyotype. Nature. 2008; 453:1064-1071. [PubMed: 18563158] 
a

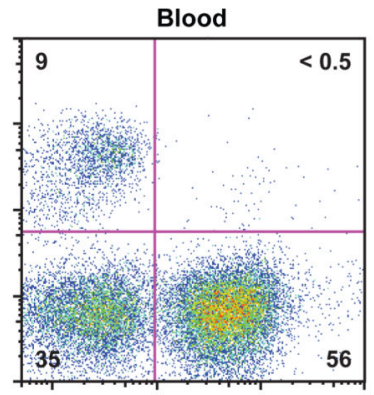

Typhlosole

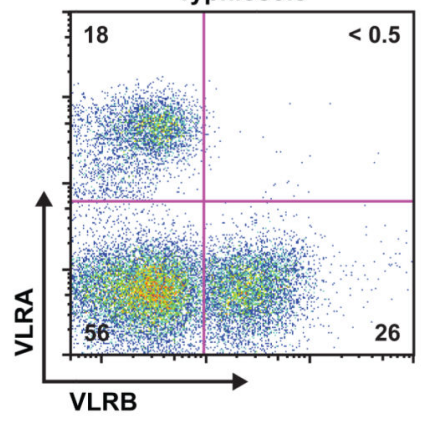

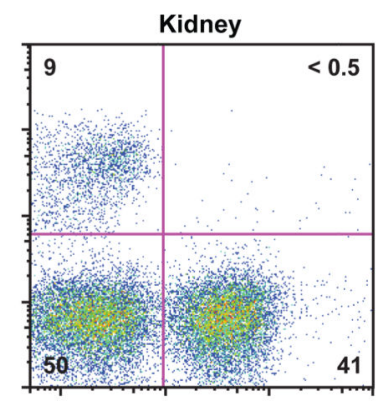

Gill

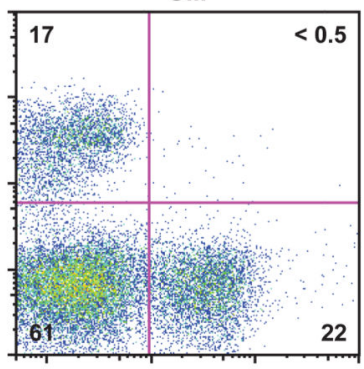

b
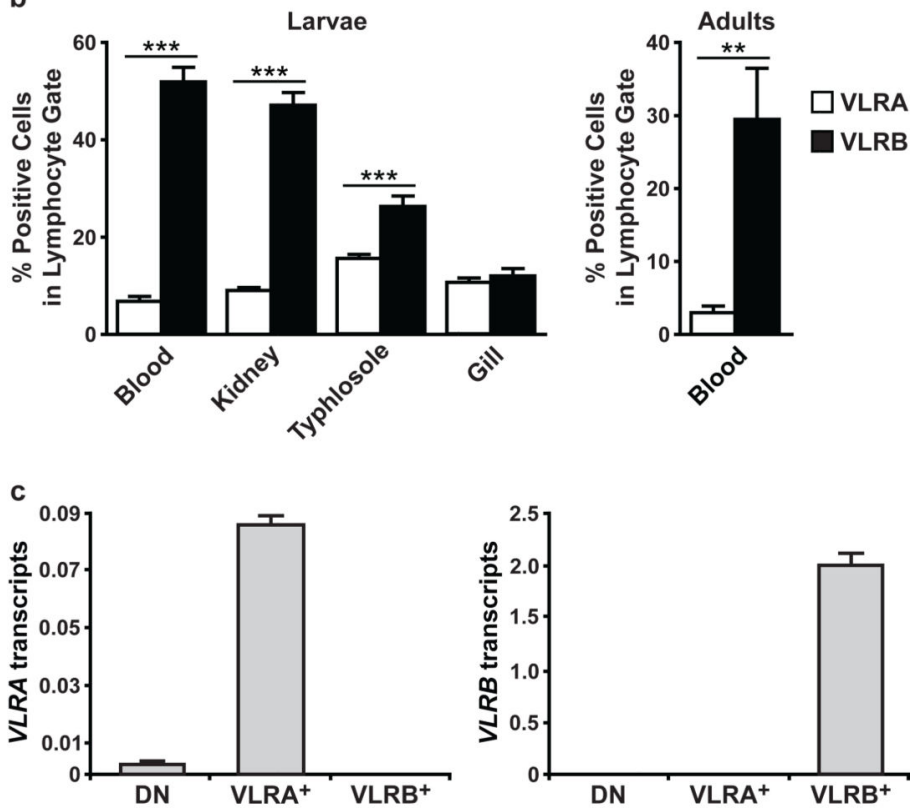

Figure 1. VLRA and VLRB expression define distinct lymphocyte populations a, FACS analysis of lymphocyte-gated cells stained with anti-VLRA (R110) and anti-VLRB (4C4) antibodies. b, Percentages of VLRA and VLRB expressing lymphocytes in lamprey tissues. Larvae (left panel) $n=23$, except gill $n=6$; adults (right panel) $n=8$ (*** $P<0.001$; $* * P<0.01)$. c, Lymphocyte-gated cells were stained as in (a) and separated based on surface expression of VLRA and VLRB by fluorescence-activated cell sorting (FACS). Sorted cells were analyzed for VLRA (left) and VLRB (right) transcripts by quantitative real-time PCR (qPCR), $n=3$. Error bars indicate s.e.m. 
a
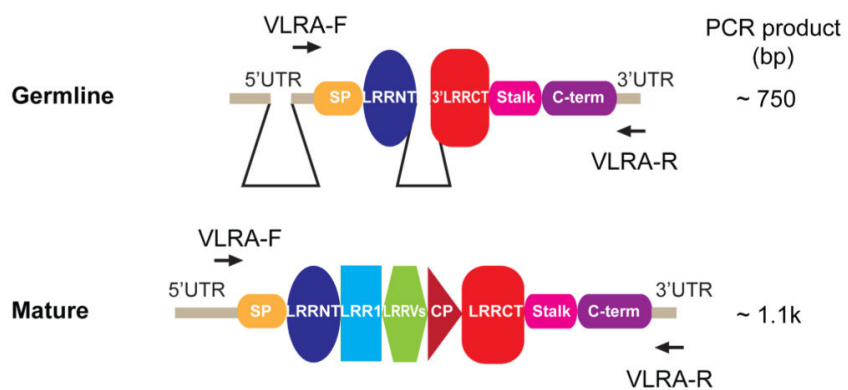
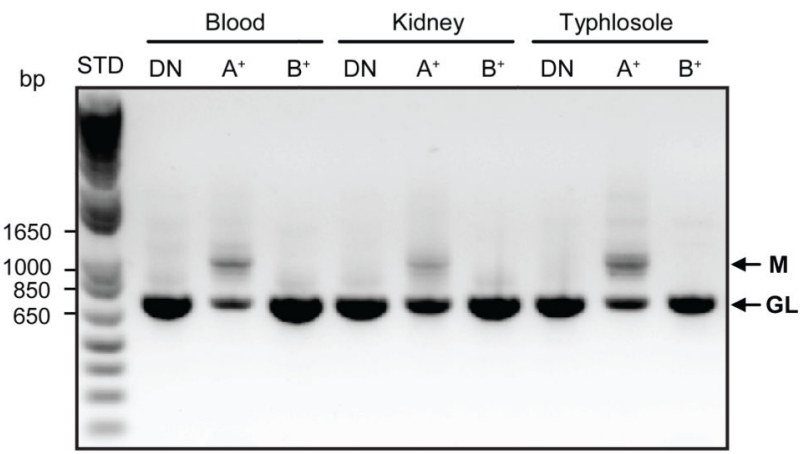

b
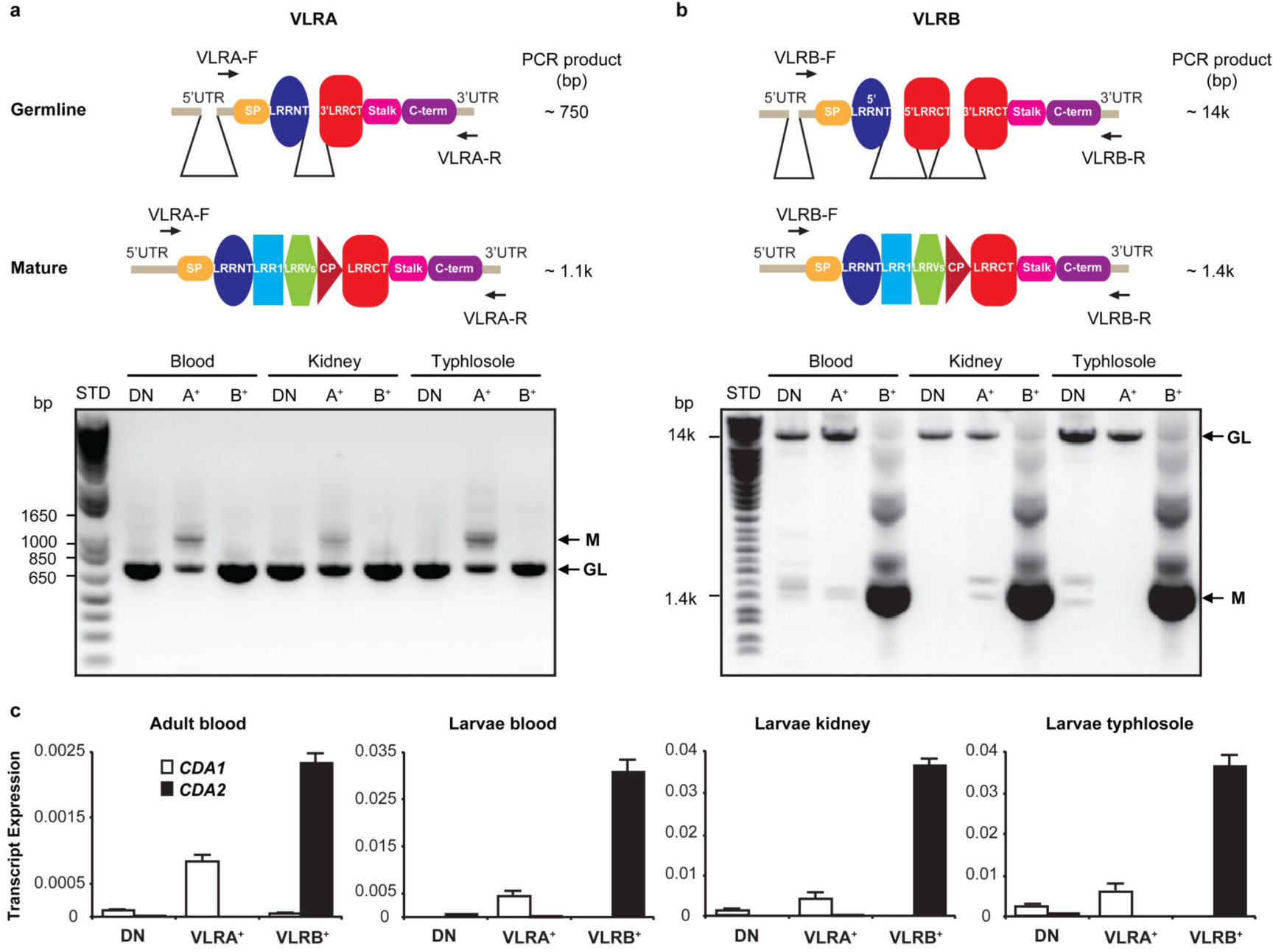

Larvae blood

Larvae kidney

Larvae typhlosole
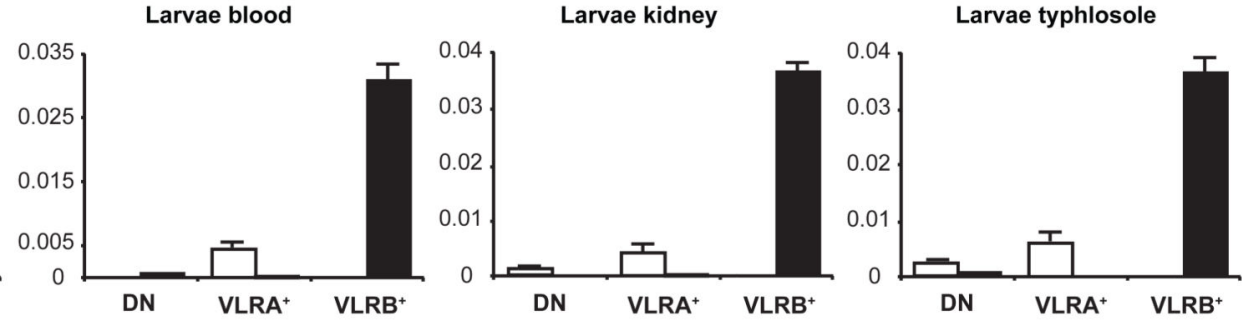

Figure 2. Monoallelic assembly of VLRA and VLRB genes

$\mathbf{a}, \mathbf{b}$, Schematic of VLRA (a) and VLRB (b) genes before (top panel) and after (middle panel) gene assembly. Forward and reverse primer locations and predicted sizes of PCR products are indicated. Lymphocytes from the indicated tissues were stained with antiVLRA (R110) and anti-VLR-B (4C4) antibodies and lymphocyte-gated cells were FACS sorted into three populations: VLRA $-/ \mathrm{VLRB}^{-}(\mathrm{DN}), \mathrm{VLRA}^{+}\left(\mathrm{A}^{+}\right)$, and $\mathrm{VLRB}^{+}\left(\mathrm{B}^{+}\right) . V L R s$ were amplified from genomic DNA of the sorted lymphocyte populations (bottom panel). Germ-line (GL) and mature (M) products were verified by sequence analysis of representative DNA clones. c, CDA1 and CDA2 expression in sorted lymphocytes was measured by QPCR. Error bars indicate s.e.m., $n=3$. 


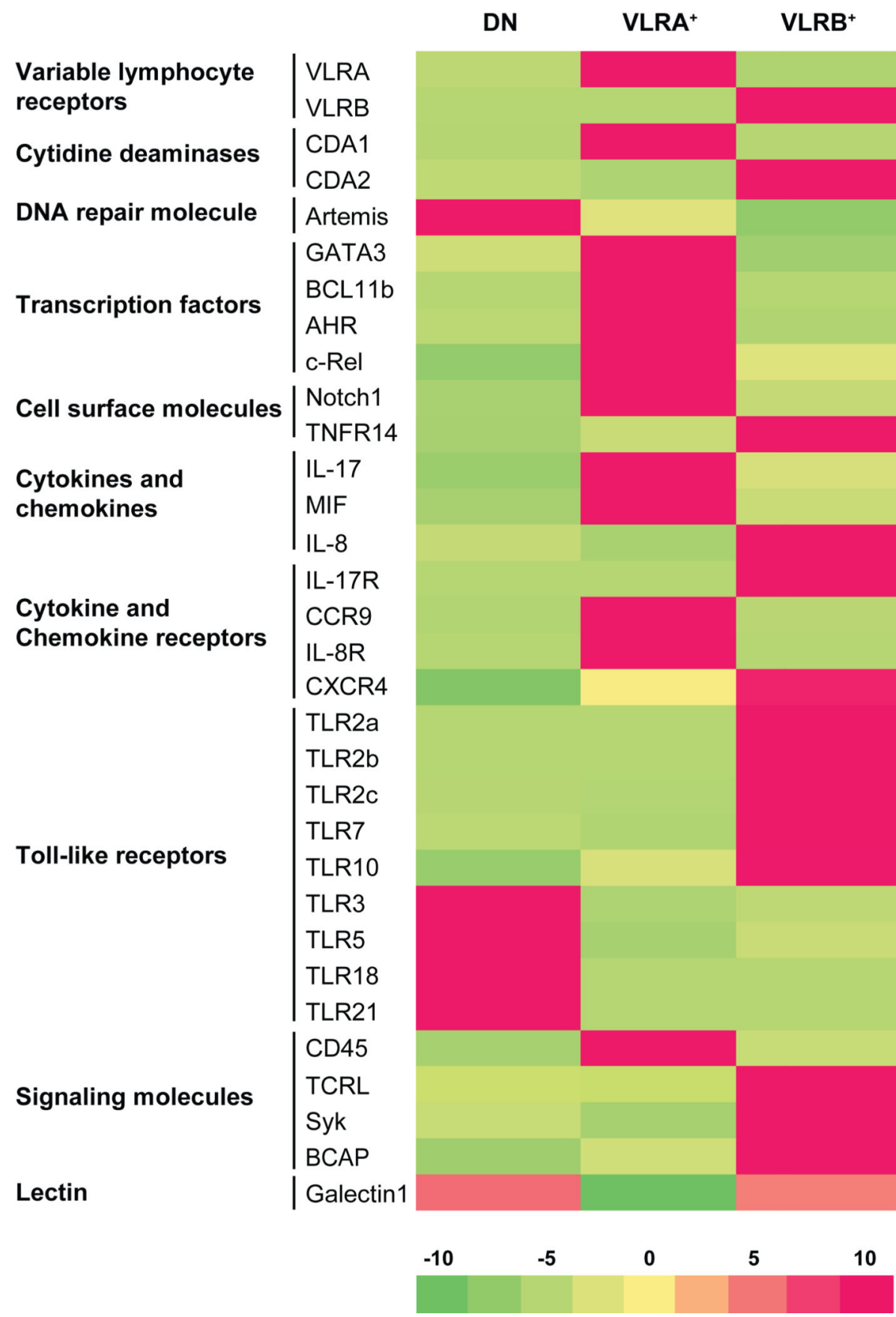

Figure 3. Differential gene expression profiles of VLRA ${ }^{+}$and $\mathrm{VLRB}^{+}$lymphocytes Lamprey lymphocytes were FACS sorted into three populations on the basis of VLRA and VLRB surface expression. Relative transcript levels of the indicated genes were measured by QPCR and compiled into a heat map as described in Methods. 
a

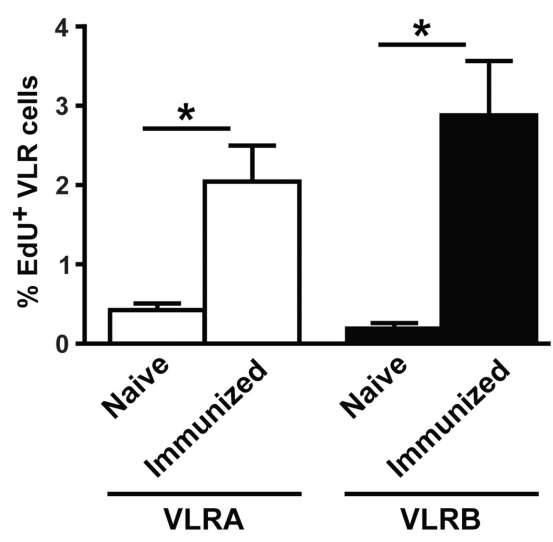

C

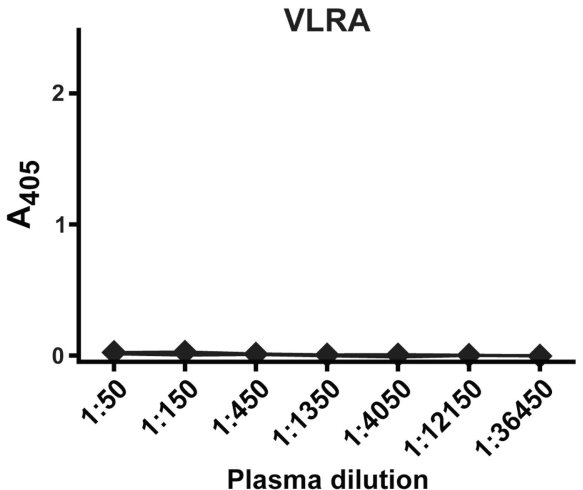

b

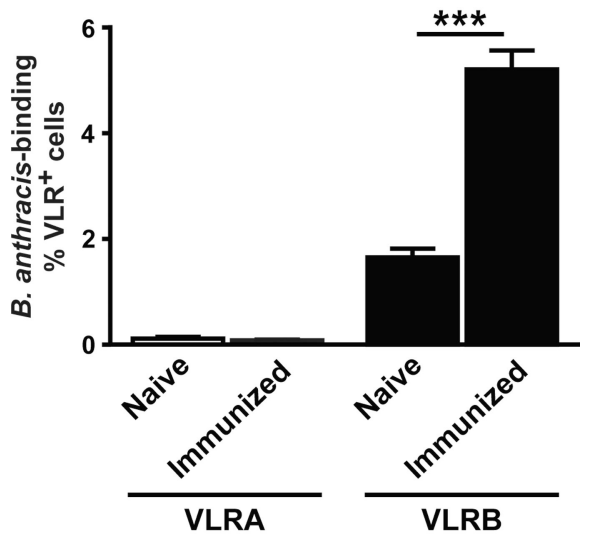

VLRB

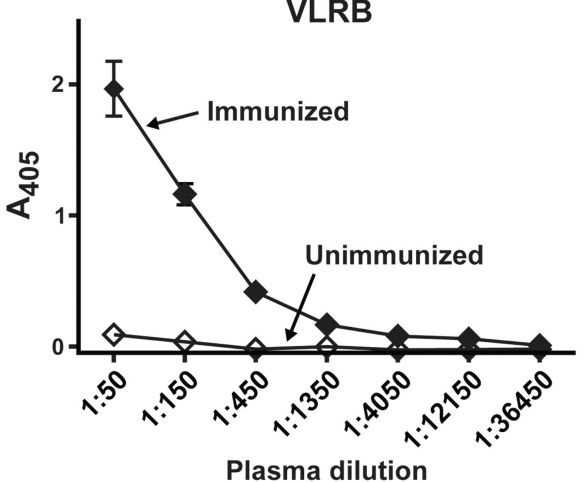

Plasma dilution

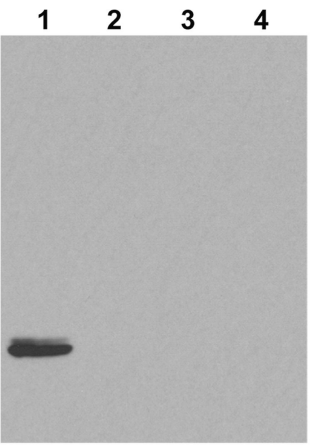

anti-VLRA (9B3)

Figure 4. Antigen-activated VLRA ${ }^{+}$lymphocytes do not secrete their receptors

a, Immunization with $B$. anthracis exosporium induces proliferation of $\mathrm{VLRA}^{+}$and $\mathrm{VLRB}^{+}$ lymphocyte $(n=5 ; * P<0.05)$. b, VLRA ${ }^{+}$lymphocytes do not bind to $B$. anthracis spores before immunization or 14 days after booster immunization $(n=5$; *** $P<0.001)$. c,d, VLRB, but not VLRA, is secreted. c, $B$. anthracis-specific VLRA and VLRB reactivity was evaluated by ELISA with anti-VLRA (9A6) and anti-VLRB (4C4) mAbs. d, Immunoblot of VLRA (lane 1) and VLRB (lane 2) HEK-293T cell transfectant lysates, naïve lamprey 
plasma (lane 3), and immunized plasma (lane 4) under reducing conditions. R110 = antiVLRA rabbit antiserum; 9B3 = anti-VLRA mAb. 


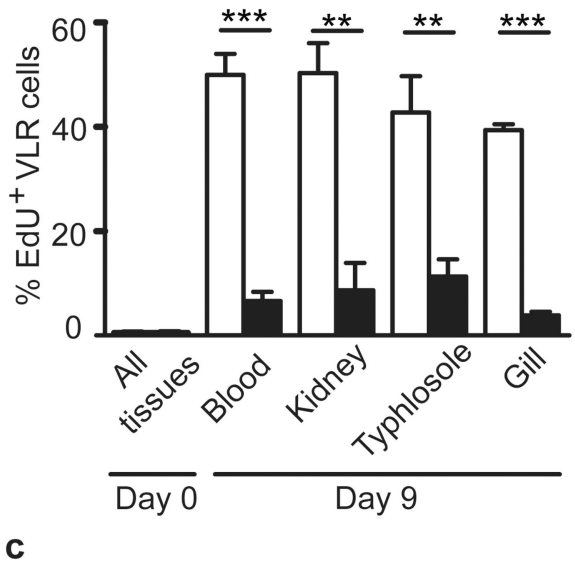

b

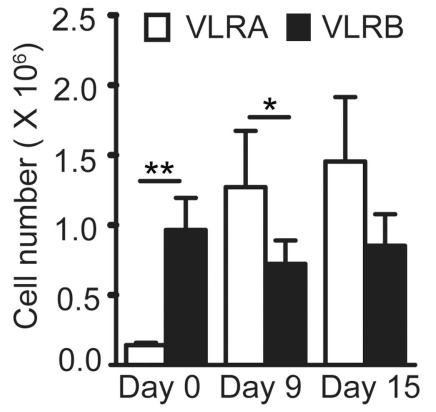

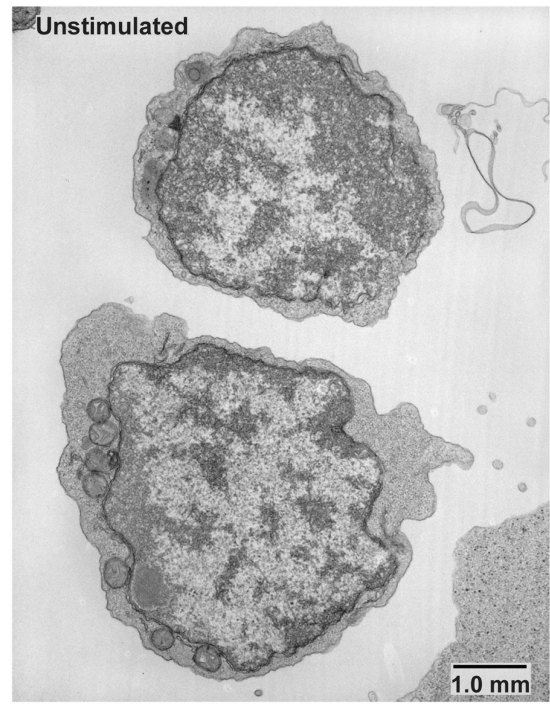

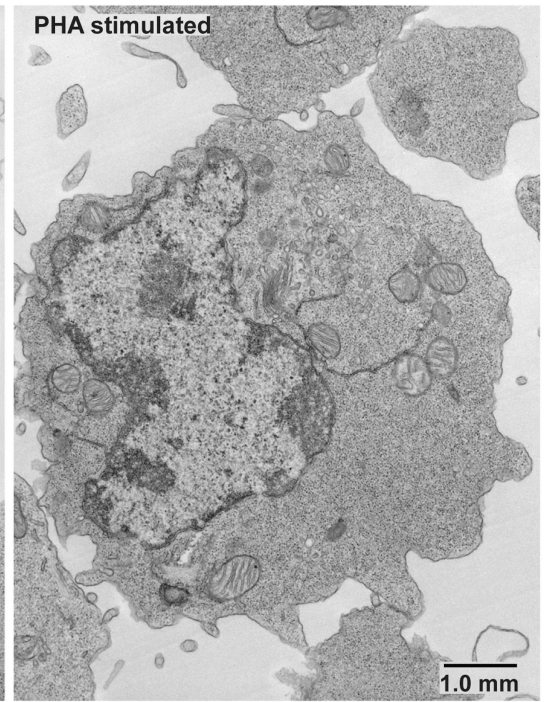

d

$\square$ Unstimulated

PHA stimulated
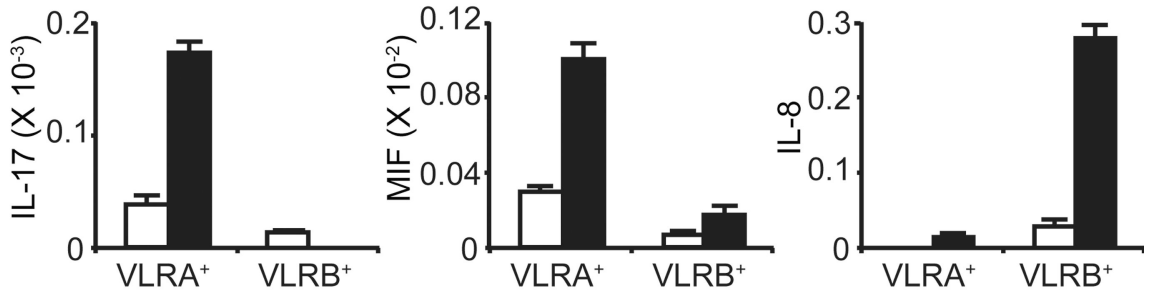

Figure 5. PHA preferentially stimulates VLRA ${ }^{+}$lymphocytes

$\mathbf{a , b}$, In vivo PHA stimulation. Lampreys were given $25 \mu \mathrm{g}$ of PHA by intracoelomic injection on day 0 . Proliferation (a) and absolute numbers (b) of $\mathrm{VLRA}^{+}$and $\mathrm{VLRB}^{+}$cells were determined on days 9 and 15 after injection; (a) $n=4$; (b) $n=7$; *P< 0.05 ; ** $P<$ 0.01 ; $* * * P<0.001$ ). c, Transmission electron microscopy imaging of unstimulated (left panel) and PHA stimulated (day 9) (right panel) $\mathrm{VLRA}^{+}$cells. PHA stimulated cells have a larger diameter [naïve mean $=3.8 \pm 0.1 \mu \mathrm{m}(n=7)$ versus PHA stimulated mean $=7.4 \pm 0.3 \mu \mathrm{m}$ $(n=7, P<0.01)]$ and increased cytoplasm. d, Cytokine expression by $\mathrm{VLRA}^{+}$and $\mathrm{VLRB}^{+}$ 
lymphocytes. FACS sorted blood lymphocytes were isolated from PHA stimulated (day 7, $\mathrm{n}$ $=3$ ) or unstimulated lampreys $(\mathrm{n}=3)$ and analyzed for expression of IL-17 (left panel), MIF (middle panel), and IL-8 (right panel) by QPCR. Error bars indicate s.e.m. 